\title{
Finite-temperature chiral transition in QCD with quarks in the fundamental and adjoint representation.
}

\section{Francesco Basile}

Dipartimento di Fisica dell'Università di Pisa and INFN,

Via Buonarroti 2, 56127 Pisa, Italy. E-mail: basileesns . it

\section{Andrea Pelissetto}

Dipartimento di Fisica dell'Università di Roma "La Sapienza” and INFN,

Piazzale Moro 2, 00185 Roma, Italy. E-mail: Andrea.Pelissetto@roma1.infn. it

\section{Ettore Vicari*}

Dipartimento di Fisica dell'Università di Pisa and INFN,

Via Buonarroti 2, 56127 Pisa, Italy. E-mail: vicari@df . unipi.it

\begin{abstract}
We study the nature of the finite-temperature chiral transition in QCD with $N_{f}$ light quarks in the fundamental and adjoint representation. Universality and renormalization-group (RG) arguments show that the possibility of having a continuous transition is related to the existence of a stable fixed point (FP) in the RG flow of a 3D Landau-Ginzburg-Wilson $\Phi^{4}$ theory with the same chiral symmetry-breaking pattern. The RG flow of these theories is studied by field-theoretical approaches, computing and analyzing high-order perturbative series, up to six loops. According to this RG analysis, the transition in QCD can be continuous only for $N_{f}=2$. In this case it belongs to the $3 \mathrm{D} \mathrm{O}(4)$ universality class. We also find a stable FP corresponding to a 3D universality class with symmetry breaking $\mathrm{U}(2)_{L} \otimes \mathrm{U}(2)_{R} \rightarrow \mathrm{U}(2)_{V}$, which implies that the transition can be continuous also if the axial-anomaly effects are suppressed at $T_{c}$. In the case of quarks in the adjoint representation, we can have a continuous transition for $N_{f}=1,2$. For $N_{f}=1$ it belongs to the $\mathrm{O}(3)$ universality class. For $N_{f}=2$ it belongs to a new 3D universality class characterized by the symmetry breaking $\mathrm{SU}(4) \rightarrow \mathrm{SO}(4)$.
\end{abstract}

XXIIIrd International Symposium on Lattice Field Theory

25-30 July 2005

Trinity College, Dublin, Ireland

${ }^{*}$ Speaker. 
The thermodynamics of Quantum Chromodynamics (QCD) is characterized by a transition from a low- $T$ hadronic phase, in which chiral symmetry is broken, to a high- $T$ phase with deconfi ned quarks and gluons (quark-gluon plasma), in which chiral symmetry is restored [1]. Our understanding of the fi nite- $T$ phase transition is essentially based on the relevant symmetry and symmetry-breaking pattern (SBP). In the presence of $N_{f}$ light quarks the relevant symmetry is the chiral symmetry $\mathrm{U}(1)_{V} \otimes \mathrm{SU}\left(N_{f}\right)_{L} \otimes \mathrm{SU}\left(N_{f}\right)_{R}$. At $T=0$ this symmetry is spontaneously broken to $\mathrm{U}(1)_{V} \otimes \mathrm{SU}\left(N_{f}\right)_{V}$ with a nonzero quark condensate $\langle\bar{\psi} \psi\rangle$. The fi nite- $T$ transition is related to the restoring of the chiral symmetry. It is therefore characterized by an $N_{f} \times N_{f}$ complex-matrix order parameter $\Phi_{i j}$, related to the bilinear quark operator $\bar{\psi}_{L i} \psi_{R j}$, and the SBP

$$
\mathrm{SU}\left(N_{f}\right)_{L} \otimes \mathrm{SU}\left(N_{f}\right)_{R} \rightarrow \mathrm{SU}\left(N_{f}\right)_{V}
$$

If the axial $\mathrm{U}(1)_{A}$ symmetry is effectively restored at $T_{c}$, the expected SBP becomes

$$
\mathrm{U}\left(N_{f}\right)_{L} \otimes \mathrm{U}\left(N_{f}\right)_{R} \rightarrow \mathrm{U}\left(N_{f}\right)_{V}
$$

Lattice Monte Carlo (MC) simulations suggest that these is not the case in three-color QCD. However, since the anomaly gets suppressed in the large- $N_{c} \operatorname{limit}\left(\partial_{\mu} J_{5}^{\mu} \propto \frac{1}{N_{c}} Q\right), \operatorname{SBP}(2)$ may be relevant in the large- $N_{c}$ limit.

Although deconfi nement and chiral symmetry restoration are apparently related to different mechanisms, they seem to be somehow coupled in QCD, since lattice computations show that the Polyakov loop has a sharp increase at $T_{c}$ where the chiral condensate vanishes. However, the interplay between the two effects is not clear yet. Insight into this question may be gained by investigating related models, such as $\mathrm{SU}\left(N_{c}\right)$ gauge theories with $N_{f}$ Dirac fermions in the adjoint representation (aQCD). ${ }^{1}$ Unlike QCD, aQCD is also invariant under global $\mathbb{Z}_{N_{c}}$ transformations related to the center of the gauge group $\mathrm{SU}\left(N_{c}\right)$, as in pure $\mathrm{SU}\left(N_{c}\right)$ gauge theories. There are two well-defi ned order parameters in the light-quark regime, related to the confi ning and chiral modes, i.e. the Polyakov loop and the quark condensate. One generally expects two transitions: a deconfi nement transition at $T_{d}$ associated with the breaking of the $\mathbb{Z}_{N_{c}}$ symmetry, and a chiral transition at $T_{c}$ in which chiral symmetry is restored. In aQCD with $N_{f}$ massless fhoors the chiralsymmetry group extends to $\mathrm{SU}\left(2 N_{f}\right)$. At $T=0$ this symmetry is expected to spontaneously break to $\mathrm{SO}\left(2 N_{f}\right)$, due to quark condensation. Therefore the SBP at the finite- $T$ chiral transition is expected to be

$$
\mathrm{SU}\left(2 N_{f}\right) \rightarrow \mathrm{SO}\left(2 N_{f}\right)
$$

with a symmetric $2 N_{f} \times 2 N_{f}$ complex matrix as order parameter related to the bilinear quark condensate. If the axial $\mathrm{U}(1)_{A}$ symmetry is restored at $T_{c}$, the SBP is

$$
\mathrm{U}\left(2 N_{f}\right) \rightarrow \mathrm{O}\left(2 N_{f}\right)
$$

MC simulations for $N_{c}=3$ and $N_{f}=2[2,3]$ show that the deconfi nement transition at $T_{d}$ is fi rst order. Results at the chiral transition appear consistent with a continuous transition. Interestingly, the ratio between the two critical temperatures turns out to be quite large, $T_{c} / T_{d} \approx 8$, suggesting a rather weak interplay between the corresponding underlying mechanisms.

\footnotetext{
${ }^{1}$ AQCD is asymptotically free only for $N_{f}<11 / 4$, thus only the cases $N_{f}=1,2$ are interesting.
} 
In order to study the nature of the fi nite- $T$ chiral transition in QCD and $\mathrm{QQCD}$, we exploit universality and renormalization-group (RG) arguments, as originally applied by Pisarski and Wilczek in Ref. [4]. They can be summarized as follows.

(i) Let us fi rst assume that the phase transition at $T_{c}$ is continuous for vanishing quark masses. In this case the length scale of the critical modes diverges approaching $T_{c}$, becoming eventually much larger than $1 / T_{c}$, which is the size of the euclidean "temporal" dimension at $T_{c}$. Therefore, the asymptotic critical behavior must be associated with a 3D universality class with the same SBP.

(ii) The existence of such a 3D universality class can be investigated by considering the most general Landau-Ginzburg-Wilson (LGW) $\Phi^{4}$ theory compatible with the given SBP, which describes the critical modes at $T_{C}$. Neglecting the $\mathrm{U}(1)_{A}$ anomaly, it is given by

$$
\mathscr{L}_{\mathrm{U}(N)}=\operatorname{Tr}\left(\partial_{\mu} \Phi^{\dagger}\right)\left(\partial_{\mu} \Phi\right)+r \operatorname{Tr} \Phi^{\dagger} \Phi+\frac{u_{0}}{4}\left(\operatorname{Tr} \Phi^{\dagger} \Phi\right)^{2}+\frac{v_{0}}{4} \operatorname{Tr}\left(\Phi^{\dagger} \Phi\right)^{2} .
$$

If $\Phi_{i j}$ is a generic $N \times N$ complex matrix, the symmetry is $\mathrm{U}(N)_{L} \otimes \mathrm{U}(N)_{R}$, which breaks to $\mathrm{U}(N)_{V}$ if $v_{0}>0$, thus providing the LGW theory relevant for QCD with $N_{f}=N$. If $\Phi_{i j}$ is also symmetric, the global symmetry is $\mathrm{U}(N)$, which breaks to $\mathrm{O}(N)$ if $v_{0}>0$, which is the case relevant for aQCD with $N_{f}=N / 2$. The reduction of the symmetry to $\mathrm{SU}\left(N_{f}\right)_{L} \otimes \mathrm{SU}\left(N_{f}\right)_{R}$ for QCD $\left[\mathrm{SU}\left(2 N_{f}\right)\right.$ for aQCD], due to the axial anomaly, is achieved by adding determinant terms, such as

$$
\mathscr{L}_{\mathrm{SU}(N)}=\mathscr{L}_{\mathrm{U}(N)}+w_{0}\left(\operatorname{det} \Phi^{\dagger}+\operatorname{det} \Phi\right) .
$$

Nonvanishing quark masses can be accounted for by adding an external-fi eld term $\operatorname{Tr}(H \Phi+$ h.c. $)$. (iii) The critical behavior at a continuous transition is determined by the fi xed points (FPs) of the RG fbw: the absence of a stable FP generally implies fi rst-order transitions. Therefore, a necessary condition of consistency with the initial hypothesis (i) of a continuous transition is the existence of stable FP in the corresponding LGW $\Phi^{4}$ theory. If no stable FP exists, the fi nite- $T$ chiral transition of QCD (aQCD) is predicted to be fi rst order. If a stable FP exists, the transition can be continuous, and its universal critical behavior is determined by the FP; but this does not exclude a fi rst-order transition if the system is outside the attraction domain of the stable FP.

The above arguments show that the nature of the fi nite- $T$ transition in QCD and aQCD can be investigated by studying the RG fbw of the corresponding 3D LGW $\Phi^{4}$ theories. For this purpose we consider two different fi eld-theoretical (FT) perturbative approaches. One is defi ned within the massive (disordered) phase using a zero-momentum renormalization (MZM) scheme. The other one is defi ned within the massless (critical) theory using a minimal subtraction $\overline{(\mathrm{MS}}$ ) scheme (we consider a 3D- $\overline{\mathrm{MS}}$ scheme without $\varepsilon$ expansion). The RG fbw is determined by the FPs, which are given by the common zeroes of the $\beta$-functions associated with the quartic couplings. A FP is stable if all eigenvalues of its stability matrix have positive real part. Using symbolic manipulations programs, we computed the expansion up to six loops in the MZM scheme (which requires the calculation of approximately 1500 Feynman diagrams) and up to fi ve loops in the $\overline{\mathrm{MS}}$ scheme. Since perturbative FT expansions are asymptotic, it is necessary to resum the series. This is done by exploiting Borel summability and knowledge of the large-order behavior, which is inferred by semiclassical calculations of instanton solutions. We fi rst resum the $\beta$-functions, and then search for their common zeroes. This computation is essentially nonperturbative, because the resummation uses nonperturbative information on their large-order behavior. The comparison of the analyses 
of the MZM and 3D- $\overline{\mathrm{MS}}$ expansions provides nontrivial crosschecks of the results. Details of these calculations can be found in Refs. [5, 6] (see also [7]). In the following we summarize the main results.

For $N=1$, the case relevant to $N_{f}=1 \mathrm{QCD}, \mathscr{L}_{\mathrm{U}(1)}$ reduces to the $\mathrm{O}(2)$ symmetric $\Phi^{4}$ theory, corresponding to the XY universality class of superfluid transition in ${ }^{4} \mathrm{He}$, see, e.g., [8]. The determinant term related to the axial anomaly, cf. Eq. (6), plays the role of an external fi eld, thus no continuous transition is expected, but a crossover.

The case $N=2$ without anomaly, cf. Eq. (5), relevant for $N_{f}=2 \mathrm{QCD}$, was originally analyzed by Pisarski and Wilczek [4] within the $\varepsilon \equiv 4-D$ expansion to one-loop order, which means close to $4 \mathrm{D}$. No stable FP is found close to $4 \mathrm{D}$, as also in the case of symmetric matrix fi eld relevant for $N_{f}=1 \mathrm{aQCD}$. Thus a naive extrapolation to 3D indicates fi rst-order transitions. However, in some physically interesting cases the extrapolation of $\varepsilon$-expansion calculations to $\varepsilon=1$ fails to provide the correct physical picture in 3D: for example, in the Ginzburg-Landau model of superconductors [10], and in $\mathrm{O}(2) \otimes \mathrm{O}(N)$ theories of some frustrated spin models [11]. Actually, this happens also in the case of the LGW $\Phi^{4}$ theory (5) with $N=2$. Indeed, the analysis of the high-order series shows the presence of stable FPs in both MZM and 3D-MS schemes, contradicting earlier analyses based on the $\varepsilon$ expansion around $4 \mathrm{D} .^{2}$ These results imply the existence of 3D universality classes with $\mathrm{SBP} \mathrm{U}(2)_{L} \otimes \mathrm{U}(2)_{R} \rightarrow \mathrm{U}(2)_{V}$ and $\mathrm{U}(2) \rightarrow \mathrm{O}(2),{ }^{3}$ corresponding respectively to $N_{f}=2 \mathrm{QCD}$ and $N_{f}=1 \mathrm{aQCD}$ in the case of suppressed $\mathrm{U}(1)_{A}$ anomaly.

In two-flavor QCD, taking into account the $\mathrm{U}(1)_{A}$ anomaly, SBP (1) becomes equivalent to the one of the $\mathrm{O}(4)$ vector universality class, i.e. $\mathrm{SO}(4) \rightarrow \mathrm{SO}(3)$. SBP (3) of $N_{f}=1$ aQCD is instead equivalent to the one of the $\mathrm{O}(3)$ vector universality class. This means that, if the transition is continuous, it must show the $\mathrm{O}(4)$ scaling behavior in $N_{f}=2$ QCD and the $\mathrm{O}(3)$ one in $N_{f}=1$ aQCD. See, e.g., Ref. [9] for a recent review on the $\mathrm{O}(N)$ universality classes. Actually, the LGW $\Phi^{4}$ theory corresponding to these cases is

$$
\begin{aligned}
& \mathscr{L}_{\mathrm{SU}(2)}=\operatorname{Tr}\left(\partial_{\mu} \Phi^{\dagger}\right)\left(\partial_{\mu} \Phi\right)+r \operatorname{Tr} \Phi^{\dagger} \Phi+\frac{u_{0}}{4}\left(\operatorname{Tr} \Phi^{\dagger} \Phi\right)^{2}+\frac{v_{0}}{4} \operatorname{Tr}\left(\Phi^{\dagger} \Phi\right)^{2} \\
& +w_{0}\left(\operatorname{det} \Phi^{\dagger}+\operatorname{det} \Phi\right)+\frac{x_{0}}{4}\left(\operatorname{Tr} \Phi^{\dagger} \Phi\right)\left(\operatorname{det} \Phi^{\dagger}+\operatorname{det} \Phi\right)+\frac{y_{0}}{4}\left[\left(\operatorname{det} \Phi^{\dagger}\right)^{2}+(\operatorname{det} \Phi)^{2}\right],
\end{aligned}
$$

where $w_{0}, x_{0}, y_{0} \sim g$ and $g$ parametrizes the effective breaking of the $\mathrm{U}(1)_{A}$ symmetry. If the anomaly is suppressed $(g=0)$, then $w_{0}=x_{0}=y_{0}=0 . \mathscr{L}_{\mathrm{SU}(2)}$ contains two quadratic (mass) terms, therefore it describes several transition lines in the $T$ - $g$ plane, which meet at a multicritical point for $g=0$. In the case of QCD the multicritical behavior is controlled by the $\mathrm{U}(2)_{L} \otimes \mathrm{U}(2)_{R}$ symmetric theory. Possible phase diagrams in the $T-g$ plane are shown in Fig. 1 . When $g \neq 0$ the transition may be fi rst order or continuous in the $\mathrm{O}(4)$ universality class. Actually, we may also have a mean-fi eld behavior (apart from logarithms) for particular values of $g$, see Fig. 1. A similar scenario applies also to $N_{f}=1$ aQCD.

No stable FPs are found for $N>2$ in the LGW theory (5). Thus, neglecting the anomaly, transitions are always fi rst order when $N_{f}>2$ for QCD and $N_{f}>1$ for aQCD. In most cases this result does not change if we take into account the axial anomaly, cf. Eq. (6). The only exception is

\footnotetext{
${ }^{2}$ This result was overlooked in Ref. [6]. Some details can be found in Ref. [5].

${ }^{3}$ The $\mathrm{U}(2) / \mathrm{O}(2)$ universality class is also the one of the normal-to-planar superflid transition in ${ }^{3} \mathrm{He}[12,5]$.
} 

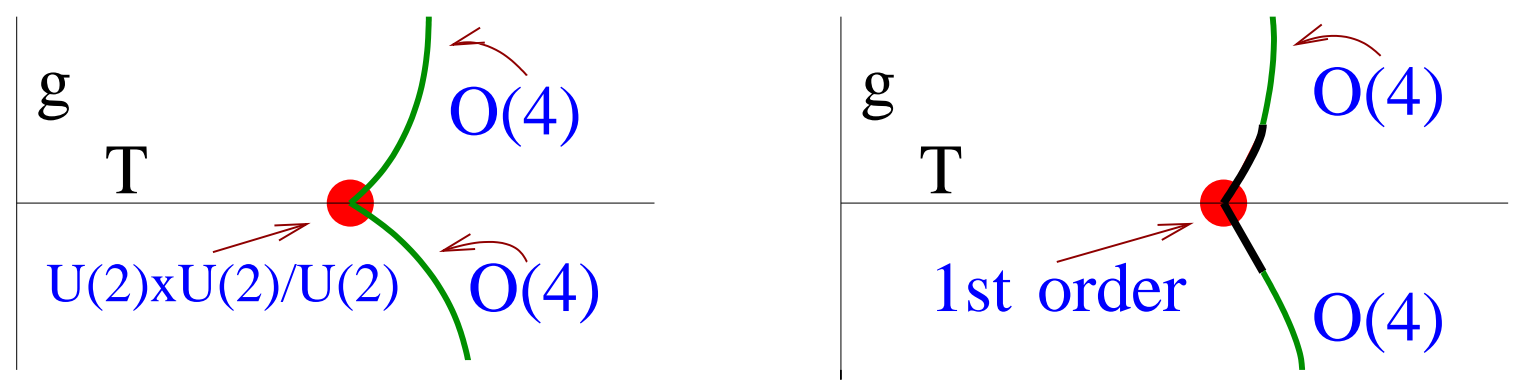

Figure 1: Possible phase diagrams in the $T-g$ plane for the LGW theory (7) describing the transition of $N_{f}=2 \mathrm{QCD}$, in the case the transition at the multicritical point, i.e. for $g=0$, is continuous (left) or first order (right). Thick black lines indicate first-order transitions. At their end points, thus for particular values of $g$, the transition should be of mean-field type (apart from logarithms).

\begin{tabular}{ccc}
\hline \hline & $\mathrm{U}(1)_{A}$ anomaly & suppressed anomaly at $T_{c}$ \\
\hline $\mathrm{QCD}$ & $\mathrm{SU}\left(N_{f}\right)_{L} \otimes \mathrm{SU}\left(N_{f}\right)_{R} \rightarrow \mathrm{SU}\left(N_{f}\right)_{V}$ & $\mathrm{U}\left(N_{f}\right)_{L} \otimes \mathrm{U}\left(N_{f}\right)_{R} \rightarrow \mathrm{U}\left(N_{f}\right)_{V}$ \\
\hline$N_{f}=1$ & crossover or fi rst order & $\mathrm{O}(2)$ or fi rst order \\
$N_{f}=2$ & $\mathrm{O}(4)$ or fi rst order & $\mathrm{U}(2)_{L} \otimes \mathrm{U}(2)_{R} / \mathrm{U}(2)_{V}$ or fi rst order \\
$N_{f} \geq 3$ & fi rst order & fi rst order \\
\hline \hline $\mathrm{aQCD}$ & $\mathrm{SU}\left(2 N_{f}\right) \rightarrow \mathrm{SO}\left(2 N_{f}\right)$ & $\mathrm{U}\left(2 N_{f}\right) \rightarrow \mathrm{O}\left(2 N_{f}\right)$ \\
\hline$N_{f}=1$ & $\mathrm{O}(3)$ or fi rst order & $\mathrm{U}(2) / \mathrm{O}(2)$ or fi rst order \\
$N_{f}=2$ & $\mathrm{SU}(4) / \mathrm{SO}(4)$ or fi rst order & fi rst order \\
\hline \hline
\end{tabular}

Table 1: Summary of the RG predictions. For each case we report the possible types of transition, indicating the universality class when the transition can also be continuous.

the case related to the $N_{f}=2 \mathrm{aQCD}$, where we fi nd a stable FP corresponding to a $3 \mathrm{D} \mathrm{SU}(4) / \mathrm{SO}(4)$ universality class, with critical exponents $v \approx 1.1$ and $\eta \approx 0.2$. Note that, although $\mathrm{SU}(4) \simeq \mathrm{O}(6)$, the $\mathrm{SBP}$ of $N_{f}=2 \mathrm{aQCD}$, i.e. $\mathrm{SU}(4) \rightarrow \mathrm{SO}(4)$, differs from that of the $\mathrm{O}(6)$ vector model, i.e. $\mathrm{SO}(6) \rightarrow \mathrm{SO}(5)$. Thus, according to the standard paradigm that relates the universality class to the SBP, the corresponding universality classes and critical behaviors must be different.

The predictions of our RG analysis for the fi nite- $T$ chiral transitions in QCD and aQCD are summarized in Table 1. These transitions have also been investigated by lattice MC simulations. Overall, MC results for two-fhvor QCD seem to favor a continuous transition in the continuum limit. However, a satisfactory check of the $\mathrm{O}(4)$ scaling behavior has not been achieved yet. Results obtained using Wilson fermions appear consistent with a continuous transition in the $\mathrm{O}(4)$ universality class [13]; MC simulations using staggered fermions appear more problematic $[14,15,16,17,3]$. Unlike $N_{f}=2 \mathrm{QCD}$, the transition scenario appears settled for $N_{f} \geq 3$ : MC simulations $[14,18]$ show fi rst-order transitions, in agreement with the RG predictions. Finally, in the case of $N_{f}=2$ aQCD the available MC results [2,3] favor a continuous transitions. But they are not yet suffi ciently precise to check the critical behavior of the $3 \mathrm{D} \mathrm{SU}(4) / \mathrm{SO}(4)$ universality class. 


\section{References}

[1] See, e.g., F. Wilczek, QCD in extreme conditions, hep-ph/0003183; F. Karsch, Lectures on Quark Matter, hep-lat/0106019.

[2] F. Karsch and M. Lütgemeier, Deconfinement and chiral symmetry restoration in an SU(3) gauge theory with adjoint fermions, Nucl. Phys. B550 (1999) 449 [hep-lat/9812023].

[3] J. Engels, S. Holtmann, and T. Schulze, Scaling and Goldstone effects in a QCD with two flavours of adjoint quarks, Nucl. Phys. $\mathbf{B 7 2 4}$ (2005) 357 [hep-lat/ 0505008 ]; The chiral transition of $N_{f}=2$ QCD with fundamental and adjoint fermions, PoS (LAT2005) 148 [hep-lat/ 0509010 ].

[4] R.D. Pisarski and F. Wilczek, Remarks on the chiral phase transition in chromodynamics, Phys. Rev. D29 (1984) 338.

[5] F. Basile, A. Pelissetto, and E. Vicari, The finite-temperature chiral transition in $Q C D$ with adjoint fermions, JHEP 02 (2005) 044 [hep-th / 041202 ].

[6] A. Butti, A. Pelissetto, and E. Vicari, On the nature of the finite-temperature transition in QCD, JHEP 08 (2003) 029 [hep-ph / 0307036$].$

[7] P. Calabrese and P. Parruccini, Five-loop $\varepsilon$ expansion for $U(n) \otimes U(m)$ models: finite-temperature phase transition in light QCD, JHEP 05 (2004) 018 [hep-ph/ 0403140$].$

[8] M. Campostrini, M. Hasenbusch, A. Pelissetto, P. Rossi, and E. Vicari, Critical behavior of the three-dimensional XY universality class, Phys. Rev. B 63 (2001) 214503 [cond-mat / 0 010360].

[9] A. Pelissetto and E. Vicari, Critical phenomena and renormalization-group theory, Phys. Rep. 368 (2002) 549 [cond-mat/ 0012164$]$.

[10] S. Mo, J. Hove, and A. Sudbø, Order of the metal-to-superconductor transition, Phys. Rev. $\mathbf{B 6 5}$ (2002) 104501 [cond-mat/0109260].

[11] P. Calabrese, P. Parruccini, A. Pelissetto, and E. Vicari, Critical behavior of $O(2) \otimes O(N)$ symmetric models, Phys. Rev. B70 (2004) 174439 [cond-mat / 0405667 ].

[12] M. De Prato, A. Pelissetto, and E. Vicari, Normal-to-planar superfluid transition in ${ }^{3} H e$, Phys. Rev. B70 (2004) 214519 [cond-mat / 0312362 ].

[13] A. Ali Khan et al. (CP-PACS Collaboration), Phase structure and critical temperature of two-flavor $Q C D$ with renormalization-group improved gauge action and clover improved Wilson action, Phys. Rev. D63 (2001) 034502 [hep-lat/ 0008011 ].

[14] F. Karsch, E. Laermann, and A. Peikert, Quark mass and flavor dependence of the QCD phase transition, Nucl. Phys. B605 (2001) 579 [hep-lat / 0012023$].$

[15] J. B. Kogut and D. K. Sinclair, Scaling behavior at the $N_{t}=6$ chiral phase transition for 2-flavor lattice QCD with massless staggered quarks and an irrelevant 4-fermion interaction, Phys. Rev. D64 (2001) 034508 [hep-lat/0104011].

[16] J. Engels, S. Holtmann, T. Mendes, and T. Schulze, Finite-size-scaling behavior for $3 d O(4)$ and $O(2)$ spin models and QCD, Phys. Lett. B514 (2001) 299 [hep-lat/ 0105028].

[17] M. D'Elia, A. Di Giacomo, and C. Pica, Two flavor QCD and Confinement, hep-lat/ 0503030.

[18] Y. Iwasaki, K. Kanaya, S. Sakai, and T. Yoshié, Chiral phase transition in lattice QCD with Wilson quarks, Z. Physik C71 (1996) 337 [hep-lat/ 9504019 ]. 